\title{
Modifying locally the safety profile to improve the confinement of magnetic field lines in tokamak plasmas
}

\author{
D. Constantinescu ${ }^{1}$ and M.-C. Firpo ${ }^{2}$ \\ ${ }^{1}$ Dept of Applied Mathematics, Association Euratom-MECI, University of \\ Craiova, Craiova 200585, Romania \\ ${ }^{2}$ Laboratoire de Physique des Plasmas CNRS-Ecole Polytechnique, 91128 \\ Palaiseau cedex, France
}

\begin{abstract}
Using Hamiltonian models for the magnetic field lines, we propose a methodology to improve their confinement through the creation of transport barriers. A local modification of the safety profile creating a low shear zone is shown to be sufficient to locally enhance drastically the regularity of the magnetic field lines without requesting a reversed shear. The optimal benefits of low shear are obtained when the value $q_{0}$ of the safety profile in the low shear zone is sufficiently far from the main resonance values $m / n$ with low $m$ and $n$, in the case of large enough values of those $(m, n)$ mode amplitudes. A practical implementation in tokamak plasmas should involve electron cyclotron current drive to locally modify the magnetic shear.
\end{abstract}

PACS numbers: $52.55 . \mathrm{Fa}, 05.45 .+\mathrm{a}, 52.25 . \mathrm{Gj}$

Published in Nuclear Fusion 52 (2012) 054006. 


\section{Introduction}

\subsection{Motivations}

Improving confinement in fusion tokamak plasmas is one of the critically important issues for the achievement of a viable fusion reactor. During the last two decades, internal transport barriers (ITBs) have enabled to increase the energy confinement time through the creation of regions of the tokamak plasma core in which the local ion and electron thermal diffusivities are substantially reduced, nearly down to their neoclassical values [1]. There is nowadays a large amount of experimental evidence revealing the importance of the shape of the $q$-profile in conditioning the existence of ITBs in tokamaks. Decisive ingredients were given notably by the experimental results obtained by Eriksson et al. [2] who demonstrated experimentally that the $q$-profile could be used as a single control parameter to obtain ITBs. In these experiments, they could obtain ITBs for non-monotonic $q$-profiles but not with (strictly) monotonously growing $q$, while other parameters such as the $\mathbf{E} \times \mathbf{B}$ flow shear were kept the same. Additional evidence was later given by Sauter et al [3] through a dedicated experiment. They controlled the $q$-profile using inductive current to generate positive and negative current density perturbations in the plasma center, with negligible input power. In this way, they were able to demonstrate that the electron confinement could be modified significantly solely by perturbing the current density profile. Other experiments in various tokamaks showed that having an inner region with low magnetic shear was indeed sufficient to obtain ITBs $[4,1]$. In particular, in the case of Razumova et al observations [4], the formation of electron ITBs was correlated to some local flattening of the otherwise monotonously growing $q$-profile, that is to the creation of a low-shear region. In the present work, our interest will be put in this later case.

The fact that the $q$-profile can be used as a single control parameter to obtain ITBs is an invitation to focus on a strongly simplified picture of tokamak plasmas, namely on a purely magnetic approach, and to address the zero-order problem of the stochasticity and confinement of magnetic field lines. We believe that this approach is fully justified to model electron stochastic transport and ITBs, since electrons have very small Larmor radii. This simplified picture of the plasma confinement problem is obviously limited, as it is non self-consistent and does not involve possible nondeterministic and/or intermittent turbulence effects.

\subsection{Hamiltonian models for magnetic field lines}

As it is well known, the equations of the magnetic field lines in a toroidal system may be written in the following Hamiltonian form $[5,6]$

$$
\frac{d \theta}{d \varphi}=\frac{\partial \psi_{p}}{\partial \psi_{t}}, \frac{d \psi_{t}}{d \varphi}=-\frac{\partial \psi_{p}}{\partial \theta}
$$

where the magnetic coordinates are used: the poloidal $\psi_{p}$ and toroidal $\psi_{t}$ magnetic fluxes, the poloidal and toroidal angles $(\theta, \varphi)$. In this representation $\theta$ and $\psi \equiv \psi_{t}$ are canonically conjugated and $\psi_{p}$ acts as an Hamiltonian function. Generically, this is a one-and-a-half Hamiltonian system

$$
\psi_{p}(\theta, \psi, \varphi)=\int W(\psi) d \psi+\varepsilon \sum_{m, n} \psi_{p_{m, n}}^{(1)}(\psi) \cos \left(m \theta-n \varphi+\chi_{m n}\right)
$$

where one has introduced the winding profile $W(\psi)=q(\psi)^{-1}$ that is the inverse of the safety factor. The first term of the r.h.s. is the poloidal flux associated to the 
axisymmetric equilibrium determined by the safety factor $q(\psi)$ and the second term accounts for the effects of magnetic perturbations, that may be external (ripple) or intrinsic (MHD). In the following, the toroidal flux $\psi$ will be normalized to its edge maximum value, so that the toroidal flux will be comprised between the values 0 and 1, corresponding respectively to the magnetic axis and to the edge.

In order to compute the magnetic field line trajectories, a computationally low cost solution lies in the use of maps. Let us here introduce the generic form of a symmetric Poincaré map

$S M_{\varepsilon}:\left\{\begin{array}{l}\bar{\theta}=\left(\theta+2 \pi W(X)+\frac{\varepsilon}{2} \frac{\partial S}{\partial X}(\theta, X)+\frac{\varepsilon}{2} \frac{\partial S}{\partial X}(\bar{\theta}, X)\right)(\bmod 2 \pi) \\ \bar{\psi}=\psi-\frac{\varepsilon}{2} \frac{\partial S}{\partial \theta}(\theta, X)-\frac{\varepsilon}{2} \frac{\partial S}{\partial \bar{\theta}}(\bar{\theta}, X)\end{array}\right.$

where $X=X(\theta, \psi) \in D$ is the unique solution of the implicit equation

$$
X=\psi-\frac{\varepsilon}{2} \frac{\partial S}{\partial \theta}(\theta, X) .
$$

In the above expressions, $S(\theta, X)$ denotes the generating function of the system restricted to the poloidal section $\varphi=0[7]$.

Definition The system (1) defined from the Hamiltonian (2) has the twist property if $W^{\prime}(\psi) \neq 0$ for all $\psi \in[0 ; 1]$. It is a nontwist system if there exists $\psi_{0} \in(0 ; 1)$ such that $W^{\prime}\left(\psi_{0}\right)=0$ and $W(\psi)$ has an extremum in $\psi_{0}$. It is a degenerate twist system if there exists $\psi_{0} \in(0 ; 1)$ such that $W^{\prime}\left(\psi_{0}\right)=0$ and $W(\psi)$ is a monotonous function.

For twist systems, KAM or Aubray-Mather theories or Greene's criterion can be applied to characterize transport barriers using the discrete system generated by the Poincaré map [8]. The non-twist systems have been also intensively studied $[9,10,11,12,13,14,15,16,17]$ (see also the references [5] and [18]). It was proved that a robust transport barrier (formed by many invariant tori, corresponding to invariant circles in the discrete system) exists in the low shear region. In this case, the transport barrier intersects the curve where the twist condition is violated, known as the shearless curve. The degenerate twist systems did not attract as much attention. They may however have important applications in fusion plasma physics related e.g. to the above mentioned special properties of low shear, yet monotonous, safety profiles in favoring transport barriers and to the H-mode improved scenario.

\section{Creating a transport barrier through a local modification of the safety profile}

\subsection{The methodology}

Proposition 2.1 If the map $S M_{\varepsilon}$ is compatible with toroidal geometry, and if there is $\psi_{0} \in(0 ; 1)$ such that $W^{\prime}\left(\psi_{0}\right)=0$ and $W_{0}=W\left(\psi_{0}\right) \notin \mathbb{Q}$ then, for small enough values of $\varepsilon$, the map $S M_{\varepsilon}$ has an invariant rotational circle $R C_{W_{0}}$ with the rotation number $W_{0}$. The map has also infinitely many invariant rotational circles with the rotation number close to $W_{0}$. These invariant rotational circles, situated in the neighborhood of $R C_{W_{0}}$, form a transport barrier which intersects the curve

$$
C_{\text {barrier, }, \varepsilon}: \psi=\psi_{0}+\frac{\varepsilon}{2} \frac{\partial S}{\partial \theta}\left(\theta, \psi_{0}\right) .
$$


Important remark: The reversed shear of the winding function (or of the safety profile) is not a mandatory condition for obtaining a large transport barrier formed by many neighboring invariant circles.

Consequences In tokamak plasmas having a monotonous safety factor, we can propose a method to build (electron) transport barriers in a prescribed zone by modifying locally the safety factor without affecting its monotony: it is sufficient to create a zone where locally $W^{\prime}(\psi)=0$.

A possible mathematical procedure to flatten locally the winding profile $W(\psi)$ in order to produce a transport barrier preventing the outer diffusion of magnetic field lines is the following. The controlled winding function $W_{c}(\psi)$ may depend on three values of $\psi$, namely $\psi_{1}<\psi_{0}<\psi_{2}$. It could have the following properties:

- $W_{c}$ coincides with $W$ on the intervals $\left[0 ; \psi_{1}\right]$ and $\left[\psi_{2} ; 1\right]$;

- $W_{c}$ is continuous and derivable in $\psi_{1}$ and $\psi_{2}$;

- the derivative of $W_{c}$ in $\psi_{0}$ is zero;

- $W_{c}$ is a monotonously decreasing function.

A candidate satisfying these criteria is given by

$$
W_{c}(\psi)= \begin{cases}W(\psi) & \text { for } 0 \leq \psi \leq \psi_{1} \\ W_{0}-c_{1}\left(\psi-\psi_{0}\right)^{7}-c_{2}\left(\psi-\psi_{0}\right)^{5}-c_{3}\left(\psi-\psi_{0}\right)^{3} & \text { for } \psi_{1} \leq \psi \leq \psi_{2} \\ W(\psi) & \text { for } \psi_{2} \leq \psi \leq 1\end{cases}
$$

where the coefficients $W_{0}, c_{1}, c_{2}$ and $c_{3}$ are computed using the continuity and derivability of $W_{c}$ in $\psi_{1}$ and $\psi_{2}$ (four conditions).

\subsection{Example of application for a model of magnetic field lines}

In order to demonstrate the efficiency of the proposed methodology, let us consider a model for magnetic perturbations corresponding to the symmetric tokamap case with three poloidal modes $(m=1,2$ and 3$)$. The map reads

$S M_{\varepsilon}:\left\{\begin{array}{l}\bar{\theta}=\left(\theta+2 \pi W(X)-\frac{\varepsilon}{2} \frac{1}{(1+X)^{2}} \sum_{m=1}^{3}(\cos (m \theta)+\cos (m \bar{\theta}))\right)(\bmod 2 \pi) \\ \bar{\psi}=\psi-\frac{\varepsilon}{2} \frac{X}{X+1} \sum_{m=1}^{3}(\sin (m \theta)+\sin (m \bar{\theta}))\end{array}\right.$

A -physically realistic- winding profile may be given by

$$
W(\psi)=(2-\psi)\left(2-2 \psi+\psi^{2}\right) / 4 .
$$

The magnetic perturbations are $H_{m}(\psi)=-\frac{1}{2 \pi m} \frac{\psi}{1+\psi}$, for $m \in\{1,2,3\}$. As visible on the phase space portrait drawn in Figure 1, a large chaotic zone appears for $\varepsilon=\simeq 0.0637$. The main modes $(3,2),(2,1)$ and $(3,1)$, having respectively the rotation numbers $2 / 3,1 / 2$ and $1 / 3$ can be observed around $\psi=0.25, \psi=0.45$ and $\psi=0.75$ respectively. Due to the very small value of $\varepsilon$, the perturbations influence mainly the region situated in the vicinity of these main modes. A chaotic zone appears due to the overlapping of the stable and unstable manifolds of the hyperbolic points. A contrario, magnetic field lines close to $\psi=0$ and $\psi=1$ are regular. There is no internal transport barrier within the chaotic region. Figure 2 depicts the phase portrait obtained with 


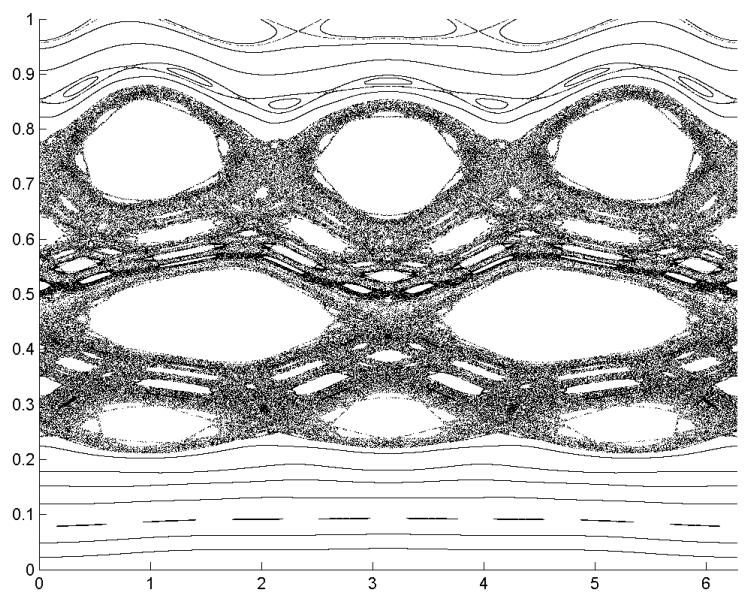

Figure 1. Phase portrait of the symmetric tokamap (3) for $\varepsilon=0.4 /(2 \pi) \simeq$ 0.0637 .

the same stochasticity parameter $\varepsilon$ as above but using the controlled winding profile $W_{c}$ with $\psi_{1}=0.3, \psi_{0}=0.4$ and $\psi_{2}=0.7$. In this case, a transport barrier is formed in the low shear region around $\psi=\psi_{0}=0.4$. The transport barrier obtained for

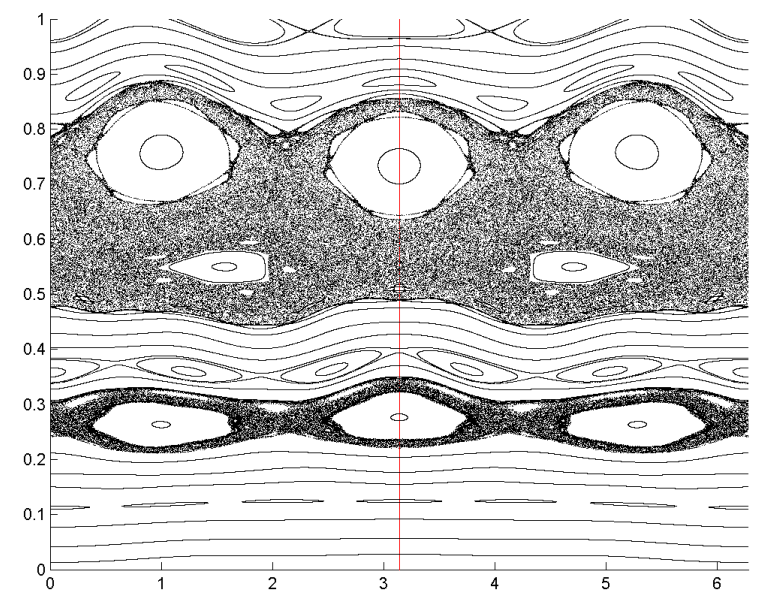

Figure 2. Phase portrait of the symmetric tokamap with three poloidal modes (3) for the controlled winding profile $W_{c}$ for the same value of $\varepsilon$ as in Figure 1. $W_{c}$ has been constructed from $W$ through the above described methodology with $\psi_{1}=0.3, \psi_{0}=0.4$ and $\psi_{2}=0.7$.

the controlled winding function corresponding to $\psi_{1}=0.3, \psi_{0}=0.6$ and $\psi_{2}=0.7$ is presented in Figure 3. Inside the transport barrier is visible the curve $C_{\text {barrier, } \varepsilon}$, the equation of which is given by $\psi=\psi_{0}+\varepsilon / 2 \psi_{0} /\left(1+\psi_{0}\right) *(\sin \theta+\sin (2 \theta)+\sin (3 \theta))$. 
Figure 4 shows the original strictly monotonously growing $q$-profile and the two

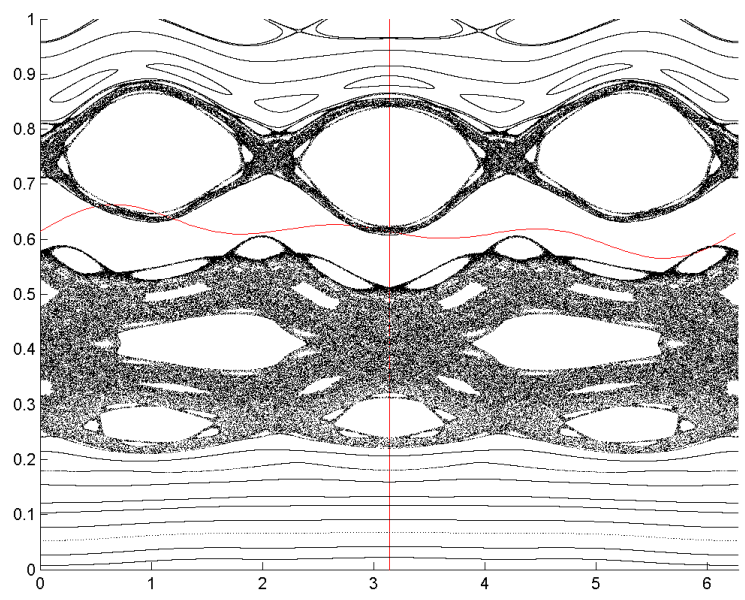

Figure 3. Phase portrait of the symmetric tokamap with three poloidal modes (3) for the controlled winding profile $W_{c}$ constructed from $W$ through the above described methodology with $\psi_{1}=0.3, \psi_{0}=0.6$ and $\psi_{2}=0.7$. The stochasticity parameter $\varepsilon$ is the same as in Figures 1 and 2, namely $\varepsilon=0.4 /(2 \pi)$. The red curve

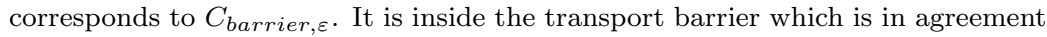
with Proposition 2.1.

controlled $q$-profiles used in Figures 2 and 3.

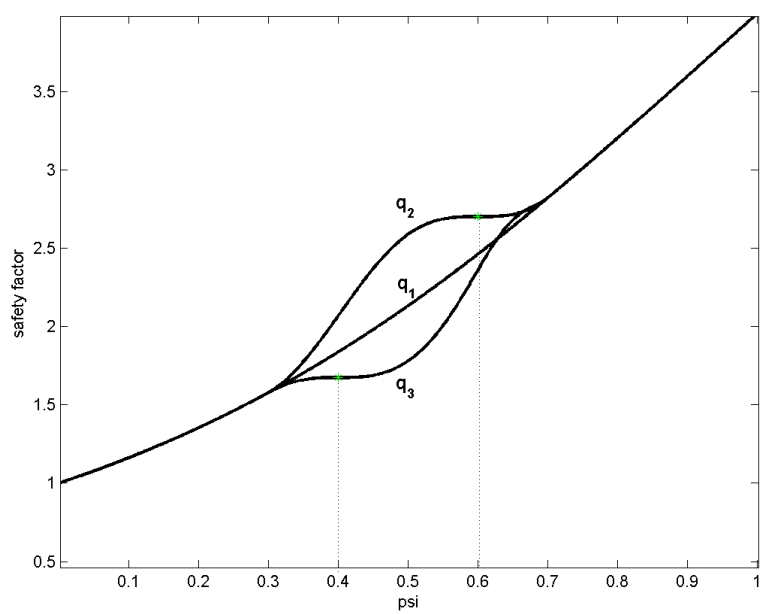

Figure 4. $q_{1}$ denotes the original $q$-profile associated to the Figure $1, q_{2}$ the modified controlled $q$-profile associated to the Figure 2 and $q_{3}$ the modified controlled $q$-profile associated to the Figure 3. 

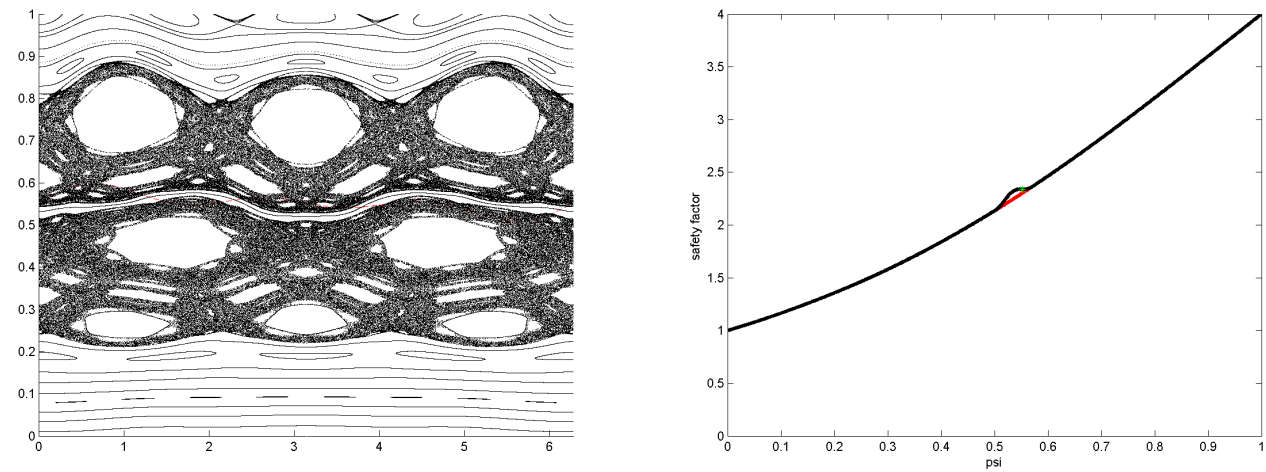

Figure 5. (Left) Phase portrait of the symmetric tokamap with three poloidal modes (3) for the controlled winding profile $W_{c}$ constructed from $W$ with $\psi_{1}=0.5, \psi_{0}=0.5525$ and $\psi_{2}=0.57$. The stochasticity parameter $\varepsilon$ is the same as in the previous figures $(\varepsilon=0.4 /(2 \pi))$. This picture should be compared with the original phase portrait given in Figure 1. One clearly observes the creation of an inner transport barrier for magnetic field lines. (Right) Original safety profile (red curve) and controlled safety profile (black curve) constructed with $\psi_{1}=0.5$, $\psi_{0}=0.5525$ and $\psi_{2}=0.57$.

The beneficial effect of the vanishing of the shear profile away from resonances is shown in Ref. [19] to be increased if the radial extent of the low-shear region is increased producing a wider inner transport barrier. In view of Figure 4, one may argue that the modification of the $q$-profile given in this example is not as local as one may wish for a realistic application of the method since $\psi_{1}$ and $\psi_{2}$ are rather far apart. These values were chosen in order to facilitate the visualization of barriers. We shall conclude this Section by considering a truly local modification of the $q$-profile. In this case, the distance between $\psi_{1}$ and $\psi_{2}$ is just 0.07 and a thinner, yet visible, transport barrier is created in the case of the controlled $q$-profile, as depicted in Figure 5.

\section{Impact of the value of the $q$-profile at the low shear region}

In Ref. [19], a detailed analysis of the influence of the $q$-profile within an Hamiltonian model for the magnetic field lines was conducted. To be specific, the $m=1$ symmetric tokamap was used with families of $q$-profiles satisfying $q>1$. The study was able to exemplify the dramatic and benefic influence of low shear, using reserved-shear or monotonous $q$-profile with an inflexion point, on magnetic confinement. However, this study was somehow biased because it ignored the possibility of resonances, namely that the value of the winding profile in the low shear region $W_{0}$ may approach the winding values of the magnetic resonances $W_{m, n} \equiv n / m$. This effect was studied in Ref. [20] for families of reversed-shear $q$-profiles. It appears that when the winding value associated to the zero shear $W_{0}$ crosses resonance values $W_{m, n}$ the confinement may deteriorate even for very small values of the stochasticity parameter, $\varepsilon$, that gives the order of magnitude of magnetic perturbations. The reason for this phenomenon is due to the fact that when $W_{0} \simeq n / m$ the system phase-space exhibits a typical non-twist pattern of island chains with a double separatrix. This effect is obviously prominent in the case of resonances with modes having small $m$ and $n$ values. A small 
amount of perturbation is sufficient to produce the stochastization and merging of both stochastic layers [21]. This phenomenon is expected to take place also for the degenerate case associated to winding (or safety) profiles monotonously growing with an inflexion point considered here.

In this respect, let us consider a modification of the same winding profile as before, as given in Eq. (4), in which the shear vanishes at $\psi_{0}=0.5$, with $q_{0}=1 / W_{c}\left(\psi_{0}\right)$ slightly below 2. The corresponding phase portrait is given in Figure 6 . In this case, there is no transport barrier around $\psi_{0}$. More than that, the benefits of low shear are no longer visible since the stochastic region is even larger than in the phase portrait corresponding to the original strictly monotonous winding profile (see Figure 1). For

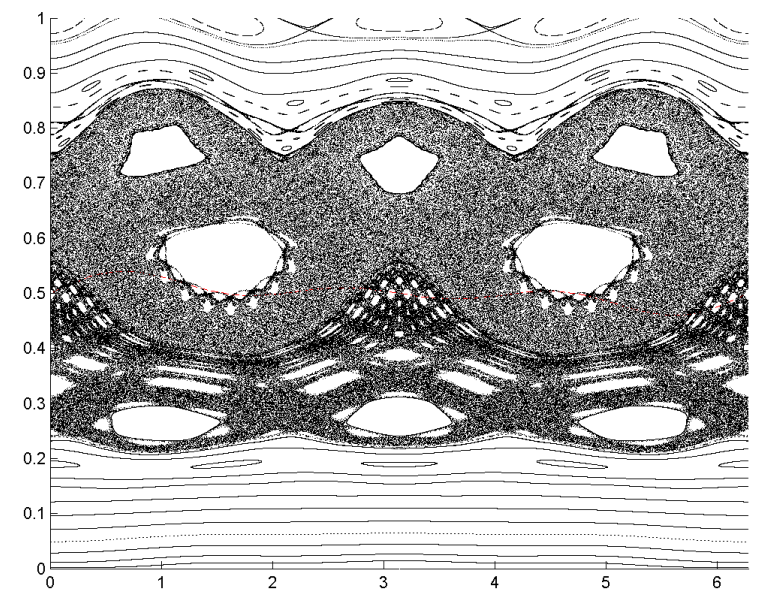

Figure 6. Phase portrait of the symmetric tokamap with three poloidal modes (3) for the controlled winding profile $W_{c}$ constructed from $W$ through the methodology exposed in part 2.1 with $\psi_{1}=0.4, \psi_{0}=0.5$ and $\psi_{2}=0.8$. The stochasticity parameter $\varepsilon$ is the same as in Figures 1,2 and 3 , namely $\varepsilon=0.4 /(2 \pi)$.

comparison, let us eventually consider another modification of the winding profile which yields a large transport barrier around $\psi_{0}$. This case is obtained for a modified winding profile with $\psi_{1}=0.2$ and $\psi_{2}=0.6$ and the associated phase portrait is plotted on Figure 7. In this case, $\psi_{0}$ has the same value as in Figure 6 but the value of the safety profile at the low shear, namely $q_{0}$, is sufficiently far from the main resonant cases given by $3 / 2,2$ and 3 . The safety factors corresponding to these last two cases are presented in Figure 8.

The results just presented are not in contradiction with Proposition 2.1 and call for a comment on its meaning. Proposition 2.1 asserts that invariant rotational circles having the irrational rotation number $W_{0}$ exist for small enough values of $\varepsilon$, say for $\varepsilon<\varepsilon_{0}$. In this Section, we observed that $\varepsilon_{0}$ is smaller in the case when $W_{0}$ is close to a main rational as $1 / 3,1 / 2,2 / 3$, than in the case when $W_{0}$ is far from them. Figures 6 and 7 show in fact that, for the same $\varepsilon=0.4 /(2 \pi)$, there is no transport barrier when $W_{0}$ is almost $1 / 2$ and that there is a large transport barrier when $W_{0}$ is far enough from $1 / 3,1 / 2$ and $2 / 3$. It means only that the $\varepsilon_{0}$ corresponding to the $W_{0}$ close to $1 / 2$ involved in Figure 6 is smaller than $0.4 /(2 \pi)$. 


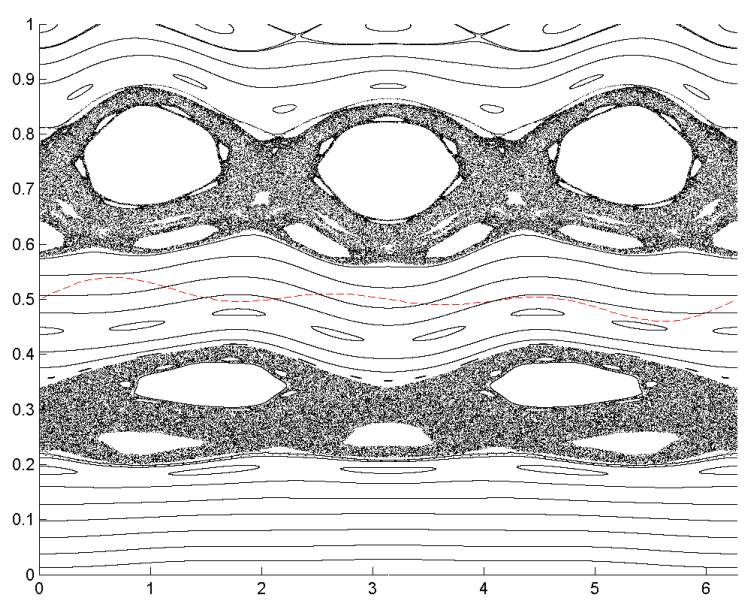

Figure 7. Phase portrait of the symmetric tokamap with three poloidal modes (3) for the controlled winding profile $W_{c}$ constructed from $W$ through the methodology exposed in part 2.1 with $\psi_{1}=0.2, \psi_{0}=0.5$ and $\psi_{2}=0.6$. The stochasticity parameter $\varepsilon$ is the same as in Figures 1,2 and 3, namely $\varepsilon=0.4 /(2 \pi)$.

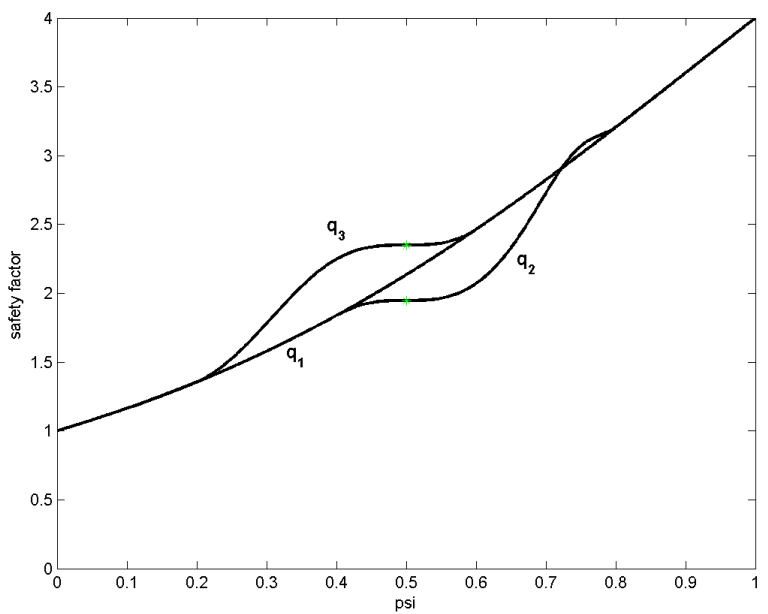

Figure 8. $q_{1}$ denotes the original $q$-profile associated to the Figure $1, q_{2}$ the modified controlled $q$-profile associated to the Figure 6 and $q_{3}$ the modified controlled $q$-profile associated to the Figure 7. 


\section{Conclusion}

In this article, using some Hamiltonian models for the magnetic field lines, we have proposed a proof of principle to enhance the confinement of magnetic field lines through a local modification of the shear of the safety profile creating a local low shear zone. A practical implementation of this methodology in tokamak plasmas would be to drive the current at the suitable position with electron cyclotron current drive (ECCD). Even if the driven current would be small in this case compared to the total current (less than a few percents), this would be sufficient to impact on the local shear.

In order to make quantitative predictions, it is now necessary to move to a more physically relevant model for magnetic perturbations. This may be provided e.g. by Abdullaev's model for internal MHD modes [7] or for external magnetic perturbations created by a set of saddle coils in tokamak plasmas [22] that require more complicate numerical implementations.

Similar studies may be applied to reverse field pinches (RFPs), that recently demonstrated improved performance related to magnetic chaos healing [23] yet through non-axisymmetric states [24, 25, 26, 27].

\section{Acknowledgments}

The authors are indebted to S.S. Abdullaev, M. Bécoulet, I. Caldas, D. del-CastilloNegrete, O. Dumbrajs, D.F. Escande, V. Igochine, M. Roberto, G. Spizzo, F. Volpe and A. Wurm for discussions and comments. The authors thank the organizers and participants of SFP2011 for creating a stimulating scientific atmosphere.

[1] J. W. Connor et al. A review of internal transport barriers physics for steady-state operation of tokamaks. Nucl. Fusion, 44:R1-R49, 2004.

[2] L.-G. Eriksson, C. Fourment, V. Fuchs, X. Litaudon, C.D. Challis, F. Crisanti, B. Esposito, X. Garbet, C. Giroud, N. Hawkes, P. Maget, D. Mazon, and G. Tresset. Discharges in the jet tokamak where the safety factor profile is identified as the critical factor for triggering internal transport barriers. Phys. Rev. Lett., 88(145001), 2002.

[3] O. Sauter, S. Coda, T. P. Goodman, M. A. Henderson, R. Behn, A. Bottino, E. Fable, An. Martynov, P. Nikkola, and C. Zucca. Inductive current density perturbations to probe electron internal transport barriers in tokamaks. Phys. Rev. Lett., 94(10):105002, Mar 2005.

[4] K. A. Razumova et al. Formation of electron transport barriers under ecr control of the $q(r)$ profile in the t-10 tokamak. Plasma Phys. Rep., 27:273, 2001.

[5] S. S. Abdullaev. Construction of Mappings for Hamiltonian Systems and Their Applications. Springer, 2006

[6] D. F. Escande, Description of magnetic field lines without arcanes, SFP2011.

[7] S. S. Abdullaev Generic magnetic field model in poloidal divertor tokamaks in the presence of resonant magnetic perturbations. Nucl. Fusion, 50:034001, 2010.

[8] R. S. McKay, Renormalisation in area-preserving maps, World Scientific Singapore, New Jersey, London, Hong Kong, 1993

[9] G. A. Oda, and I. L. Caldas. Dimerized island chains in tokamaks. Chaos, Solit. and Fract. , 5:15-23, 1995.

[10] D. del Castillo-Negrete, J. M. Greene, and P. J. Morrison. Area preserving nontwist maps: periodic orbits and transition to chaos. Physica D, 91:1-23, 1996.

[11] R. Balescu. Hamiltonian nontwist map for magnetic field lines with locally reversed shear in toroidal geometry. Phys. Rev. E, 58(3):3781-3792, 1998.

[12] D. Del-Castillo-Negrete, M.-C. Firpo. Coherent structures and self-consistent transport in a mean field Hamiltonian model. Chaos, 12(2):496-507, 2002.

[13] M. Roberto, E. C. da Silva, I. L. Caldas, R. L. Viana. Non-twist field line mappings for tokamaks with reversed magnetic shear. Braz. J. Phys. 34(4b):1759-1765, 2004. 
[14] A. Wurm, A. Apte, K. Fuchss, and P. J. Morrison. Meanders and reconnectioncollision sequences in the standard nontwist map. Chaos, 15:023108, 2005.

[15] W. Horton et al, Temperature gradient driven electron transport in NSTX and Tore Supra. Nucl. Fusion 45:976-985, 2005.

[16] I. I. Rypina, M. G. Brown, F. J. Beron-Vera, H. Koçak, M. J. Olascoaga, and I. A. Udovydchenkov. Robust transport barriers resulting from strong kolmogorov-arnold-moser stability. Phys. Rev. Lett., 98(104102), 2007.

[17] J. S. E. Portela, I. L. Caldas, and R. L. Viana. Tokamak magnetic field lines described by simple maps. The European Physical Journal - Special Topics, 165:195-210, 2008.

[18] P. J Morrison, and A. Wurm. Nontwist maps. Scholarpedia, 4(9):3551, 2009.

[19] L. Nasi and M.-C. Firpo. Enhanced confinement with increased extent of the low magnetic shear region in tokamak plasmas. Plasma Phys. Control. Fusion, 51:045006, 2009.

[20] M.-C. Firpo and D. Constantinescu. Study of the interplay between magnetic shear and resonances using Hamiltonian models for the magnetic field lines. Phys. Plasmas 18:032506 (2011).

[21] S. M. Soskin, R. Mannella, O. M. Yevtushenko, I. A. Khovanov, P. V. E. McClintock, in Hamiltonian Chaos Beyond KAM Theory, Springer, Heidelberg 2010.

[22] S. S. Abdullaev. Universal asymptotics of poloidal spectra of magnetic perturbations of saddle coils in tokamaks. Phys. Plasmas 18:020701 (2011)

[23] D. F. Escande, R. Paccagnella, S. Cappello, C. Marchetto, and F. D'Angelo. Chaos healing by separatrix disappearance and quasisingle helicity states of the reversed field pinch. Phys. Rev. Lett., 85(15):3169-3172, Oct 2000.

[24] P. Piovesan D. Terranova P. Zanca M. Zuin A. Alfier D. Bonfiglio F. Bonomo A. Canton S. Cappello L. Carraro R. Cavazzana D. F. Escande A. Fassina P. Franz M. Gobbin P. Innocente L. Marrelli R. Pasqualotto M. E. Puiatti M. Spolaore M. Valisa N. Vianello P. Martin R. Lorenzini, E. Martines, RFX mod team, and collaborators. Self-organized helical equilibria as a new paradigm for ohmically heated fusion plasmas. Nature Physics, 5:570-574, 2009.

[25] M. E. Puiatti, A. Alfier, F. Auriemma, S. Cappello, L. Carraro, R. Cavazzana, S. Dal Bello, A. Fassina, D. F. Escande, P. Franz, M. Gobbin, P. Innocente, R. Lorenzini, L. Marrelli, P. Martin, P. Piovesan, I. Predebon, F. Sattin, G. Spizzo, D. Terranova, M. Valisa, B. Zaniol, L. Zanotto, M. Zuin, M. Agostini, V. Antoni, L. Apolloni, M. Baruzzo, T. Bolzonella, D. Bonfiglio, F. Bonomo, A. Boozer, M. Brombin, A. Canton, R. Delogu, G. De Masi, E. Gaio, E. Gazza, L. Giudicotti, L. Grando, S. C. Guo, G. Manduchi, G. Marchiori, E. Martines, S. Martini, S. Menmuir, B. Momo, M. Moresco, S. Munaretto, L. Novello, R. Paccagnella, R. Pasqualotto, R. Piovan, L. Piron, A. Pizzimenti, N. Pomphrey, P. Scarin, G. Serianni, E. Spada, A. Soppelsa, S. Spagnolo, M. Spolaore, C. Taliercio, N. Vianello, A. Zamengo, and P. Zanca. Helical equilibria and magnetic structures in the reversed field pinch and analogies to the tokamak and stellarator. Plasma Physics and Controlled Fusion, 51(12), DEC 2009. 36th European-Physical-Society Conference on Plasma Physics, Sofia, Bulgaria, Jun 29-Jul 03, 2009.

[26] D. Bonfiglio, M. Veranda, S. Cappello, L. Chacón and G. Spizzo Journal of Physics: Conference Series, 260:012003, 2010.

[27] M. Veranda, S. Cappello, D. Bonfiglio, L. Chacon, D. Escande, Magnetic topology and flow in helical Reversed Field Pinch (RFP) configuration from MHD simulations, 38th EPS Conference on Plasma Physics, 27 June - 1 July 2011, Strasbourg, France, P4.028. 